\title{
List length and overlap effects in forced-choice associative recognition
}

\author{
STEVEN E. CLARK and ALDEN HORI \\ University of California, Riverside, California
}

\begin{abstract}
Two experiments examined forced-choice associative recognition for OLAP and NOLAP test conditions. OLAP test trials consist of pairs with overlapping items (e.g., AB vs. AD), whereas NOLAP test trials contain no overlapping items (e.g., AB vs. CF). Previous results show better performance for NOLAP than for OLAP tests, contrary to the predictions of global memory models. The present experiments varied list length to examine the hypothesis that the NOLAP advantage is produced by recall-like retrieval processes. The use of longer lists either eliminated (Experiment 1) or greatly reduced (Experiment 2) the NOLAP advantage. However, a reliable OLAP advantage was not obtained. Implications for models are discussed.
\end{abstract}

Current global matching models base recognition decisions on the match of the test item to memory. This match may be construed as an index of the memory strength or familiarity of the test item. Included among these models are Gillund and Shiffrin's (1984) SAM model, Murdock's (1982) theory of distributed associative memory (TODAM), Pike's (1984) matrix model, and Hintzman's (1988) MINERVA 2. One of the strengths of global matching models is that the same mechanism underlies item and associative recognition. Item recognition requires subjects to recognize whether an item or event has occurred, whereas associative recognition requires subjects to recognize that items or events have co-occurred. The focus of this paper is on associative recognition.

Clark, Hori, and Callan (1993) have shown that these models make a unique prediction for associative recognition and, moreover, they have shown that this prediction is not supported by data. They used the following procedure to show this. The subjects studied a list of word pairs, denoted $\mathrm{AB}, \mathrm{CD}, \mathrm{EF}, \mathrm{GH}, \mathrm{IJ}$, and so on, where each letter represents a word. Following this list, a forcedchoice associative recognition test was given, requiring the subjects to distinguish between intact and rearranged test pairs (e.g., $\mathrm{AB}$ vs. CF, where $\mathrm{C}$ and $\mathrm{F}$ are recombined from different study pairs). On each forced-choice trial, the subjects were presented with one intact pair and two rearranged distractor pairs, and were to identify the intact pair. The critical conditions are shown in Figure 1. On overlapping (OLAP) test trials, the items in intact and rearranged alternatives shared a common item (e.g., AB would be tested against $\mathrm{AD}$ and $\mathrm{AF}$ ). On nonoverlapping

This research was supported by a grant from the Academic Senate of the University of California, and by National Science Foundation Grant DBS 9120911. We thank Ben Murdock for helpful discussions, and Tony Lin for assistance in data collection. Correspondence should be addressed to S. E. Clark, Department of Psychology, University of California, Riverside, CA 92521 (e-mail: clark@fido.ucr.edu).
(NOLAP) trials, the test pairs did not share a common item (e.g., AB would be tested against $\mathrm{CF}$ and GJ).

Global matching models predict better performance for OLAP tests than for NOLAP tests. The following mechanism underlies this prediction. For forced-choice recognition, the familiarities of each test alternative are computed, and the test item with the highest familiarity is called old or intact. For simplicity, we will describe the basis of the predictions in terms of a two- rather than a three-alternative case (although the same mechanisms apply in the latter case). Thus, an OLAP trial would present $A B$ versus $A D$, whereas a NOLAP trial would present $A B$ versus $C F$.

For each alternative on a given test trial, the familiarity is computed. We denote these familiarities as $F(A B)$, $F(A D)$, and $F(C F)$. For each target-distractor pair, the difference in familiarities is calculated $[F(A B)-F(A D)$ for the OLAP test trial, and $F(A B)-F(C F)$ for the NOLAP test trial]. Overall, targets will be more familiar than distractors. However, over test trials, the familiarity differences would vary, and on some trials they would be negative, showing higher familiarity for a distractor than for a target, thus leading to an error.

Forced-choice recognition performance is a function of the expected difference (the mean of the difference distribution) relative to the standard deviation $(S D)$ of the difference distribution. High performance is defined by a large expected difference relative to a small $S D$ of the difference distribution.

The expectations of the difference distributions are equal for OLAP and NOLAP test trials, because the target $(\mathrm{AB})$ is identical in the two cases, and the familiarities of the rearranged distractors are also equal $[F(A D)=F(C F)]$.

Since the means of the difference distributions are equal for OLAP and NOLAP test conditions, any predicted differences in performance must be due to the $S D$ of the difference distributions. First, note that the variance of the difference distribution for any two random variables, $x$ and $y$, is given as the sum of the variance for 


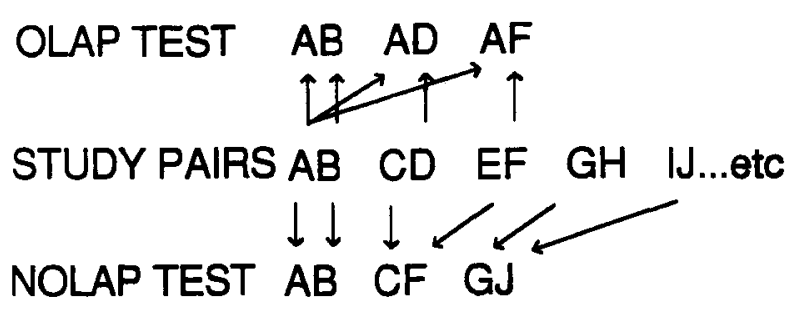

Figure 1. Design of OLAP and NOLAP test trials. Assume that words $A B, C D, E F, G H$, and $I J$ are among the pairs in a studied list. On an OLAP test trial, the test pairs overlap; for example, $A B$ is tested against $A D$ and $A F$, where Word $A$ is common to each test pair. On NOLAP test trials, however, there is no overlap of the three test pairs (AB, CF, and GJ). From "Foreed-Choice Associative Recognition: Implications for Global Memory Models," by S. E. Clark, A. Hori, \& D. E. Callan, 1993, Joumal of Experimental Psychology: Learning, Memory, \& Cognition, 19, p. 871-881. Copyright 1993 by the American Psychological Association. Adapted by permission.

$x$ plus the variance for $y$, minus twice their covariance [i.e., $\operatorname{var}(x-y)=\operatorname{var}(x)+\operatorname{var}(y)-2 \operatorname{cov}(x, y)$; see Feller, 1968, p. 236].

The key point is that on OLAP test trials, the pairs $A B$ and AD are similar due to the overlap of Item A. Thus, their familiarities will be correlated. This covariance in the familiarities reduces the $S D$ of the difference distribution for OLAP test trials relative to NOLAP test trials on which the alternatives are not similar (and thus the familiarities do not covary on a given test trial). Because the $S D$ of the difference distribution is smaller for OLAP test trials than it is for NOLAP test trials (and the mean of the difference distributions are the same), performance is predicted to be better for OLAP than for NOLAP test trials.

To illustrate these predictions, consider two different forced-choice recognition tests. Assume in each case that the difference between the mean of the target and distractor distributions is equal to 1 . First, consider the usual case, in which the target and distractor on a given test trial are selected randomly. Assuming that there is overlap in target and distractor distributions, some trials will present a distractor that is more familiar than the target, and performance will be less than perfect.

Now imagine a contrived forced-choice recognition test in which target and distractor on each forced-choice trial are "hand-picked," such that the target is always one unit more familiar than the distractor. In this case, (1) the mean of the difference distribution will be equal to 1 (just as it is when target and distractor are selected randomly); (2) since the difference is always 1 , the $S D$ of the difference distribution is 0 ; (3) the familiarities are perfectly correlated; and (4) performance will be perfect. This will be true even if there is a good deal of overlap in the target and distractor distributions.

With one exception, this prediction holds for all of the models under consideration. The exception is TODAM, which, under some conditions, can predict no difference for OLAP and NOLAP test conditions. TODAM's flexi- bility in this prediction is due to a unique property of the model-namely, that item-specific and associative information are independent.

It is important to note that the OLAP advantage is produced because the $\mathrm{AB}$ and $\mathrm{AD}$ pairs overlap at the level of the items. In all of the models except TODAM, associative recognition is based on both item and associative information. In TODAM, associations are represented as separate units, such that a given association is independent of the items that enter into the association. Because the associations are independent of the component items, and because it is the overlap of items that produces the OLAP advantage, $\mathrm{AB}$ is no more similar to $\mathrm{AD}$ than it is to CF. Thus, the familiarities of $A B$ and $A D$ are not correlated, and equal performance for OLAP and NOLAP conditions is predicted.

In TODAM, when probing memory with a word pair, some proportion of weight is given to the associative information, and some is given to the individual items. Again, since it is the overlap of items that produces the OLAP advantage, if the weight on the items is zero (i.e., if all weight is given to the associative information), TODAM will predict equal performance for OLAP and NOLAP test conditions, for the reasons described above. However, as the weight on item information is increased, an OLAP advantage is predicted.

The results of three experiments (Clark et al., 1993), however, show a reliable, and sometimes quite large, NOLAP advantage, contrary to predictions of all of the models, including TODAM. Clark (1992; Clark et al., 1993) has suggested that associative recognition is something of a hybrid of recall and recognition. Like recognition, it does not require subjects to generate individual items from memory. The test items are provided by the experimenter, and the subject needs only to judge their familiarity. However, associative recognition is like cued recall, in that it is the associative information between items that is critical to performance. Subjects must say which items occurred together. Furthermore, while it is not necessary to retrieve items from memory, subjects may do it anyway. For example, AB may be identified as intact if $A$ is used to recall $B$ (or $B$ to recall $A$ ), and $C F$ may be rejected if $C$ is used to recall $D$ (or if $F$ is used to recall $\mathrm{E}$ ). Similar recall-based recognition models have been proposed by Humphreys (1978) and Mandler (1980).

Clark et al. (1993) suggested that recall-like retrieval processes may underlie the NOLAP advantage. Their reasoning is that subjects have more cues in a NOLAP test than in an OLAP test. There are six cues on a NOLAP test trial (A, B, C, F, G, and J), but only four cues on an OLAP trial (A, B, D, and F). Thus, if items are used singly as recall cues to retrieve correct pair members, an advantage will be given to the NOLAP test condition. Nobel and Huber (1993) have confirmed this reasoning with Monte Carlo simulations using the SAM model.

The results of Clark et al. (1993) are not only inconsistent with predictions of the models, they also differ from the pattern of results shown for forced-choice item recognition. Hintzman (1988) and Tulving (1981) have 
both shown that forced-choice item recognition performance is better when the distractor is similar to the target on a given test trial than when the distractor is similar to some other item in memory. The advantage for similar distractors is parallel to the predicted advantage for OLAP test trials in associative recognition, and is predicted by the models for the same reason. When target and distractor are similar, their familiarities will be correlated.

In Tulving's (1981) experiments, the subjects studied a list of pictures, denoted A, B, C, etc. The critical forcedchoice test conditions tested either $A$ versus $A^{\prime}$ (where Picture $A^{\prime}$ is visually similar to $A$ ), or $A$ versus $B^{\prime}$ (where $B^{\prime}$ is similar to Picture $B$ from the list). The A-versus- $A^{\prime}$ case is like the OLAP condition $A B$ versus $A D$, in that the distractor is similar to the target. The A-versus- $\mathrm{B}^{\prime}$ case is like the NOLAP condition $A B$ versus $C F$, in that the distractor is similar to other information in memory, but not to the target on that particular forced-choice trial.

Hintzman (1988) presented similar test conditions to those of Tulving (1981), except that similarity was defined in terms of category membership. In the present associative recognition procedure, similarity is defined in terms of common or overlapping items across test pairs. In any case, the predictions of the models are the same: performance is predicted to be better when the distractor is similar to the target on a given forced-choice trial than when the distractor is similar to some other item (or pair) in memory. However, for item recognition, the similardistractor advantage occurs as predicted by the models, whereas the corresponding OLAP advantage in associative recognition does not.

The present paper examines a retrieval-based account of the NOLAP advantage in associative recognition. In the experiments by Clark et al. (1993), subjects studied lists that were relatively short (consisting of 28-34 word pairs). If a recall-like component produces the NOLAP advantage, studying very long lists may eliminate this effect. Assuming, as Clark et al. (1993) have suggested and Nobel and Huber (1993) have simulated, that associative recognition is based on a combination of global matching and recall, if very few items can be recalled, recognition decisions must be based primarily on global matching. In this case, if the contribution of recall is minimized and recognition is based on global matching, the NOLAP advantage should also be reduced. In fact, in the absence of recall, all global matching models, with the exception of TODAM, predict an OLAP advantage; TODAM can predict either an OLAP advantage or no difference. Thus, an OLAP advantage would be consistent with all of the global matching models, and equal performance would be consistent only with TODAM.

\section{EXPERIMENT 1}

The purpose of Experiment 1 was to examine forcedchoice associative recognition, testing with overlapping (OLAP) and nonoverlapping (NOLAP) test alternatives, while varying the study-list length. Further, previous research (Clark et al., 1993) tested using a three-alternative procedure, whereas the present experiment used a twoalternative test. Although the pattern of predictions of the global matching models is the same regardless of the number of alternatives, we wanted to verify that the NOLAP advantage is not specific to the three-alternative case. Subjects studied either a short or a long list, followed by OLAP or NOLAP test trials.

\section{Method}

Subjects. The subjects were 142 introductory psychology students from the University of California, Riverside, participating in partial fulfillment of a course requirement. There were 38 subjects in the OLAP-short-list condition, 35 in the NOLAP-short-list condition, 34 in the OLAP-long-list condition, and 35 in the NOLAPlong-list condition (data for 5, 8, 5, and 4 subjects, respectively, were not included in the analyses because those subjects' performance was at or below chance). The subjects were run in groups of 1-5.

Materials and Procedure. The subjects were presented with either a short list of 34 pairs or a long list of 100 pairs, presented at a rate of $2 \mathrm{sec} / \mathrm{pair}$. The pairs are denoted $\mathrm{AB}, \mathrm{CD}, \mathrm{EF}, \mathrm{GH}$, and so on, where each letter represents a word. The words had ratings of $50+$ occurrences/million in word-frequency norms (Kučera \& Francis, 1967; Thorndike \& Lorge, 1944). The assignment of words to pairs, as well as the order of presentation of pairs within a list, were randomized for each subject.

Prior to study, the subjects were informed that their memory for the word pairs would be tested later, and the associative nature of the test procedures was emphasized, as they were told to form associations between words within a study pair, and to avoid forming associations between different study pairs. Interactive encoding strategies, such as creating sentences or visual images from the words within a pair, were encouraged. At no time prior to study were the subjects told the nature of the test (i.e., associative recognition). After being shown the list, the subjects were given additional written instructions that described the OLAP or NOLAP test.

Following the presentation of the study list, the subjects performed a mental-arithmetic task for $45 \mathrm{sec}$, followed by a forcedchoice associative recognition test. For all test trials, one intact target and one rearranged distractor were presented, one above the other.

The test sequence consisted of either all OLAP or all NOLAP test trials. In an OLAP trial, the two test pairs overlapped. For instance, $A B$ would be tested against $A D$, where Item $A$ is common to both test pairs. For half of the trials, the common item in each pair was the left-hand member (i.e., Item $A$ in $A B$ and $A D$ ), and for the other half of the trials it was the right-hand member (i.e., Item $B$ in $A B$ and $C B$ ). In a NOLAP trial, the two test pairs did not overlap (e.g., $A B$ would be tested against $C F$ ).

In the short-list condition, 10 test trials were given, whereas in the long-list condition, 32 test trials were given. The number of test trials was the same for OLAP and NOLAP test conditions, and was constrained by the fact that a NOLAP test trial requires three study-list pairs. The position of the intact pair (i.e., top or bottom) was random for the short-list condition and counterbalanced for the long-list condition. The reason for this difference in procedure was that for the 10-trial test, the subjects might have noticed the counterbalancing, and adjusted their responses accordingly.

For both OLAP and NOLAP test sequences, a given study pair contributed to only one test trial. For example, if $A B$ and $C F$ were tested on a given NOLAP test trial, Items A, B, C, D, E, and F would not appear on another trial. This was done because if study pairs were tested on multiple test trials, the subjects could have based their decisions at the end of the test sequence on items tested or on information retrieved earlier in the test sequence.

For both long and short lists, the first two and last two study pairs were not tested. Because NOLAP test trials require more study pairs than OLAP test trials, fewer study pairs were tested. Those that were tested were selected randomly. 
Design. Test condition (i.e., OLAP or NOLAP) and list length (i.e., short or long) were manipulated between subjects.

Apparatus. Stimulus presentation and response collection were controlled by ATARI 1040ST computers, each running independently for each subject.

\section{Results and Discussion}

Percentage correct was calculated for each subject, and the averages are shown in Table 1 for all four conditions. A $2 \times 2$ analysis of variance (ANOVA) showed better recognition for the short list than for the long list $\left[F(1,138)=40.09, M S_{\mathrm{e}}=.0122, p<.001\right]$, and better performance for NOLAP than for OLAP tests, but the difference was not statistically reliable $[F(1,138)=3.37$, $\left.M S_{\mathrm{e}}=.0122, p>.07\right]$. The interaction was also significant $\left[F(1,138)=8.07, M S_{\mathrm{e}}=.0122, p<.01\right] .^{1}$

The interaction is produced in a manner consistent with the recall account of the NOLAP advantage. A separate test for the short list showed a significant NOLAP advantage $\left[F(1,71)=10.12, M S_{\mathrm{e}}=.0135, p<.01\right]$. A separate test for the long list showed that the 2\% OLAP advantage was not reliable $(F<1)$. In addition, the decrement in performance due to increasing the list length was larger for the NOLAP condition (.170) than for the OLAP condition (.065). This pattern is consistent with the proposal that the list-length increase reduces the role of recall as a source of facilitation in the NOLAP condition.

The NOLAP advantage for the short study list is consistent with previous results by Clark et al. (1993). Thus, the NOLAP advantage is not restricted to the threealternative case. For the long list, however, the NOLAP advantage was eliminated, and a small, though statistically unreliable, OLAP advantage was shown.

This pattern of results is consistent with the proposal that a long list may reduce the contribution of a recall-like process in associative recognition, so that recognition decisions would be based primarily on global matching. If subjects base their decisions solely on global matching, SAM, MINERVA 2, and the matrix model predict an OLAP advantage. The results for the long list are consistent with this proposal, although the effect was very small. However, the result should not be dismissed for this reason, because it is the only result that has not shown a NOLAP advantage.

The trend toward an OLAP advantage is consistent with all global matching models. The results of Experiment 1 are consistent with our hypothesis that increasing the list length will reduce the contribution of recall, thus

Table 1

Percentage Correct for Experiment 1

\begin{tabular}{|c|c|c|c|c|c|}
\hline \multirow[b]{3}{*}{ List Length } & \multicolumn{4}{|c|}{ Test Condition } & \multirow[b]{3}{*}{ NOLAP - OLAF } \\
\hline & \multicolumn{2}{|c|}{ OLAP } & \multicolumn{2}{|c|}{ NOLAP } & \\
\hline & $M$ & $S E$ & $M$ & $S E$ & \\
\hline Short & .779 & .019 & .866 & .020 & .087 \\
\hline Long & .714 & .018 & .696 & .018 & -.018 \\
\hline Short - Long & .065 & & .170 & & \\
\hline
\end{tabular}

Note- $M$, mean; $S E$, standard error. reducing the NOLAP advantage. However, the results are equivocal in the sense that it is unclear whether the long-list result should be characterized as showing a small OLAP advantage (that did not reach statistical significance) or as showing equal performance between OLAP and NOLAP conditions. To show support for SAM, MINERVA 2, and the matrix model, a clear OLAP advantage must be shown.

Distinguishing between these interpretations of the long-list results is important, because the OLAP advantage is predicted by models in which item-specific information contributes to associative recognition performance, whereas equal performance for OLAP and NOLAP conditions is predicted only by TODAM, which assumes that item-specific and associative information are independent.

Because the OLAP advantage in the long-list condition was quite small, a second experiment was conducted in an attempt to obtain a more convincing result. Experiment 2 used a three-alternative test procedure, following study of a long list of 100 pairs. Assuming that recalllike processes are minimized, the models predict a larger OLAP advantage as the number of overlapping alternatives is increased, because each additional alternative increases the variance of the difference distribution more for the NOLAP condition than for the OLAP condition. Thus, the OLAP advantage shown in the long-list condition of Experiment 1 may be expanded using the threealternative procedure in Experiment 2.

A comparison of the results of Clark et al.'s (1993) Experiment 3 with the short-list results of the present Experiment 1 provides an interesting contrast to this prediction. Using a three-alternative procedure, Clark et al. showed a larger NOLAP advantage than the two-alternative procedure did in the present Experiment 1 (.14 and .09, respectively). One hypothesis is that the number of alternatives may function to expand whatever result is obtained. Just as it appears to increase the magnitude of the NOLAP advantage following study of short lists, so it may also expand the magnitude of the OLAP advantage for long lists.

\section{EXPERIMENT 2}

\section{Method}

Subjects. Sixty-five introductory psychology students from the University of California, Riverside, participated in partial fulfillment of a course requirement. There were 34 subjects in the OLAP condition and 31 ( 2 of whom were rejected because their performance was at or below chance) in the NOLAP condition.

Materials and Procedure. The materials were the same as those used in the previous experiment. The subjects studied one list, consisting of 100 word pairs, presented at a rate of $3 \mathrm{sec} /$ pair. The first two and last two study pairs were not tested.

After presentation of the list, the subjects performed mental arithmetic for $45 \mathrm{sec}$, followed by either a series of OLAP or NOLAP test trials. Each OLAP test trial consisted of one intact and two overlapping rearranged test pairs (e.g., AB, AD, and AF). NOLAP test trials presented one intact and two nonoverlapping rearranged test pairs (e.g., $\mathrm{AB}, \mathrm{CF}$, and $\mathrm{GJ}$ ). 
Table 2

Percentage Correct for Experiment 2

\begin{tabular}{|c|c|c|c|c|c|}
\hline \multirow[b]{3}{*}{ List Length } & \multicolumn{4}{|c|}{ Test Condition } & \multirow[b]{3}{*}{ NOLAP - OLAP } \\
\hline & \multicolumn{2}{|c|}{ OLAP } & \multicolumn{2}{|c|}{ NOLAP } & \\
\hline & $M$ & $S E$ & $M$ & $S E$ & \\
\hline Short* & .700 & .061 & .844 & .046 & .144 \\
\hline Long & .658 & .030 & .726 & .032 & .068 \\
\hline Short - Long & .042 & & .118 & & \\
\hline
\end{tabular}

Note- $M$, mean; $S E$, standard error. *Results from Experiment 3 of Clark et al. (1993).

Both OLAP and NOLAP test conditions consisted of 18 test trials, the maximum number of test trials possible, given 96 critical pairs, because each NOLAP test trial uses five study pairs. As in Experiment 1, the position of the common item in OLAP pairs was counterbalanced. In addition, the intact pair appeared equally often in each of the three positions (top, middle, or bottom).

Design. Test condition (OLAP vs. NOLAP) was manipulated between subjects.

\section{Results and Discussion}

Percentage correct, averaged across subjects, is shown in Table 2 for both test conditions. The 7\% NOLAP advantage for the long list was not statistically reliable $\left[F(1,63)=2.43, M S_{\mathrm{e}}=.0302, p>.13\right]$.

Additional analyses examined the position (top, middle, or bottom) of the intact pair, as well as whether the common item appeared on the left or on the right in OLAP pairs. None of these analyses showed any significant differences.

The hypothesis that a three-alternative testing procedure following a long study list would produce a larger OLAP advantage was not supported. Rather, it appears that the longer list functions to reduce the magnitude of the NOLAP advantage. The results of Clark et al.'s (1993) Experiment 3 (using a short list) are shown in Table 2 for comparison. The only difference between these experiments concerned list length. With short lists, their Experiment 3 showed a .144 NOLAP advantage, compared with the .068 NOLAP advantage shown in the present experiment. It should be noted also that the .068 advantage was not statistically significant, even though twice as many subjects participated as did in Clark et al.'s (1993) Experiment 3.

Further, the comparison of Experiment 2 with Clark et al.'s (1993) Experiment 3 shows the same pattern as the short-list-long-list comparison of Experiment 1. The present experiment showed a .118 deficit in NOLAP performance, relative to Clark et al.'s Experiment 3, and a much smaller (.042) decrease in performance for the OLAP condition. Thus, just as in Experiment 1, the decrease in performance with the increase in list length was much larger for the NOLAP condition than for the OLAP condition.

\section{GENERAL DISCUSSION}

Two experiments examined forced-choice associative recognition. The critical variable was the similarity or overlap between targets and distractors on a given forcedchoice trial. For OLAP test trials, target and distractor overlapped (e.g., AB vs. AD); for NOLAP test trials they did not overlap (e.g., AB vs. CF). In three previous experiments, Clark et al. (1993) showed better performance for NOLAP than for OLAP test conditions-a result that cannot be predicted by familiarity-based global matching models of recognition. They proposed that the NOLAP advantage is produced by the operation of a recall-like retrieval process.

In the absence of such recall processes, an OLAP advantage is predicted by all global matching models except TODAM, which can predict either an OLAP advantage or no difference. To minimize the role of recall in making recognition judgments, the present experiments used longer study lists. In Experiment 1, in which a twoalternative forced-choice procedure was used, a NOLAP advantage was shown for short lists (consistent with previous results), and a small (but statistically unreliable) OLAP advantage was shown for long lists. Experiment 2 used a three-alternative procedure, following study of a long list, and showed a small (unreliable) NOLAP advantage, which was considerably smaller than that found in previous experiments (Clark et al., 1993) using shorter lists.

The results of both experiments are consistent with the proposal that the NOLAP advantage is due to the operation of recall-like retrieval processes, which are minimized when long lists are studied. These results, however, do not offer strong support for global matching models because, while the NOLAP advantage was reduced with the increase in list length, a reliable OLAP advantage was not shown. The results are less of a problem for TODAM than for the other global matching models, because TODAM can predict equal performance for OLAP and NOLAP test conditions. It makes this prediction only if it is assumed that subjects ignore item-specific information when making associative recognition judgments. This assumption is reasonable because itemspecific information is irrelevant to the associativerecognition judgment. Whether subjects actually can ignore this irrelevant information is not known.

It should be emphasized that TODAM can make the equal-performance prediction because it represents associations in such a way that they bear no similarity to the items that enter into them (an example illustrating this point is that the concept of hot dog bears very little resemblance to the concept of $d o g$ ). This property of TODAM allows for the independent retrieval of itemspecific and associative information.

As noted at the beginning of this paper, one of the strengths of global matching models is that they account for item and associative recognition with a common matching mechanism. However, for forced-choice recognition, the pattern of results for associative recognition is quite different from that for item recognition. In five experiments (including those of Clark et al., 1993), we have not produced a reliable OLAP advantage. However, Hintzman (1988) and Tulving (1981) have both shown a consistent advantage for item recognition, when distractors are similar to targets on a given forced-choice trial. Contrary to the models' predictions, these conflicting re- 
sults indicate that associative and item recognition do not operate by the same mechanism. Moreover, the effects produced by list-length manipulations provide evidence that recall-like retrieval processes are operating in forcedchoice associative recognition.

\section{REFERENCES}

CLARK, S. E. (1992). Word frequency effects in associative and item recognition. Memory \& Cognition, 20, 231-243.

Clark, S. E., Hori, A., \& Callan, D. E. (1993). Forced-choice associative recognition: Implications for global memory models. Jour nal of Experimental Psychology: Learning, Memory, \& Cognition, 19, 871-881.

FELLER, W. (1968). An introduction to probability theory and its applications (3rd ed., Vol, 1). New York: Wiley.

Gillund, G., \& ShiFFrin, R. M. (1984). A retrieval model for both recall and recognition. Psychological Review, 91, 1-67.

Hintzman, D. L. (1988). Judgements of frequency and recognition memory in a multiple-trace memory model. Psychological Review, 95, 528-551

HUMPHREYs, M. S. (1978). Item and relational information: A case for context independent retrieval. Journal of Verbal Learning \& Verbal Behavior, 17, 175-187.

KuČERA, H., \& FRANCIS, W. N. (1967). A computational analysis of present-day American English. Providence, RI: Brown University Press.
MANDLER, G. (1980). Recognition: The judgement of previous occurrence. Psychological Review, 87, 252-271.

MURDOCK, B. B., JR. (1982). A theory for the storage and retrieval of associative information. Psychological Review, 89, 609-626.

NOBEL, P. A., \& HuBER, D. E. (1993, August). Modeling forced-choice associative recognition through a hybrid of global recognition and cued-recall. Paper presented at the 15 th annual meeting of the Cognitive Science Society, Boulder, CO.

PiKE, R. (1984). A comparison of convolution and matrix distributed memory systems. Psychological Review, 91, 281-294.

THORNDIKE, E. L., \& LORGE, I. (1944). The teacher's word book of 30,000 words. New York: Columbia University, Teachers College Press.

TULVING, E. (1981). Similarity relations in recognition. Journal of Verbal Learning \& Verbal Behavior, 20, 479-496.

\section{NOTE}

1. Because the long-list condition was followed by a longer test list than the short-list condition, the statistical analysis was also done for just the first 10 test trials in the long-list condition. This analysis showed the same pattern of results as the analysis that included all 32 test trials.

(Manuscript received May 17, 1993; revision accepted for publication September $3,1993$. 